\title{
MicroRNA-mediated susceptible poplar gene expression regulation associated with the infection of virulent Melampsora larici-populina
}

Danlei Li ${ }^{\dagger}$, Feng Wang ${ }^{* \dagger}$, Chao Wang, Li Zou, Zhiying Wang ${ }^{*}$, Qiaoli Chen, Chunyang Niu, Ruizhi Zhang, Yaming Ling and Bowen Wang

\begin{abstract}
Background: Rust caused by Melampsora larici-populina is one of the most damaging diseases of poplars. Rust is considered to be a model pathogen for genetic studies because both pathogen and host genomes are available. The poplar 'Robusta', whose general rust resistance is defeated by virulent rust E4, provides suitable host material for studies of the gene regulation involved in rust resistance/susceptibility. In this study, we investigated the microRNA-mediated susceptible poplar gene expression regulation associated with the infection of virulent rust. We were particularly interested in delineating the host-pathogen interactions with a specific focus on microRNAs (miRNAs).

Results: To study the susceptibility of poplar to M. larici-populina, small RNA (sRNA) libraries, a degradome cDNA library and digital gene expression libraries were constructed for rust-inoculated and rust-free susceptible poplar 'Robusta' leaves through high-throughput sequencing. Altogether, 12,722 regulating interactions were identified. The results delineated the framework of post-transcriptional regulation of gene expression in the susceptible poplar, which was infected by the virulent rust. The results indicated that pathogen-associated molecular patterns (PAMPs) and PAMP-triggered immunity were induced by the infection of virulent rust E4 and that miRNAs still functioned at this stage. After this stage, miRNA-regulated $R$ genes, such as TIR-NBS-LRR and CC-NBS-LRR, were not fully functional. Additionally, the rust-responsive miRNAs did not regulate the signaling component genes related to the salicylic acid pathway or the hypersensitive response.

Conclusions: We found that the defense-related post-transcriptional regulation of the susceptible poplar 'Robusta' functions normally only at the stage of PAMPs and PAMP-triggered immunity (PTI). More importantly, the miRNA-mediated post-transcriptional regulation of defense signal pathway genes were inactivated by the infection of virulent rust at the stage of effector-triggered susceptibility and during the following stages of salicylic acid and hypersensitive responses. This inactivation was the major characteristic of 'Robusta' susceptibility.
\end{abstract}

Keywords: Melampsora larici-populina, Populus, Susceptibility, microRNAs, Degradome, DGE

\footnotetext{
* Correspondence: kingsummit@126.com; nematodefungi@126.com

†Equal contributors

College of Forestry, Northeast Forestry University, Harbin 150040, China
} 


\section{Background}

Rust caused by Melampsora larici-populina is one of the most severe diseases of poplars. Rust is indigenous to Eurasia and later spread to Australia [1] and North America [2]. In the Far East, M. larici-populina infection occurs in a wide range of poplar species and hybrids and often causes severe damages $[3,4]$. It has a very complex life cycle, producing 5 spore stages during its life cycle and alternating on larch (Larix spp.).

Poplars (Populus spp.) occur naturally in most parts of the northern hemisphere from subarctic to subtropical regions [5] and are widely planted beyond their natural range in both the southern and northern hemispheres [6]. Poplars and their hybrids vary in rust resistance. Although hybrids of $P$. deltoides and $P$. nigra or $P$. trichocarpa were selected for their immunity to rust [7], breakdown of the resistance to $M$. larici-populina in some of these cultivars was detected upon the appearance of races E1 and E2 [8, 9]. Subsequently, many cultivars that had been highly resistant to rust were infected by a new race, E4 [10]. Resistance and susceptibility are at opposite ends of the same spectrum, and research on disease resistance cannot be conducted without reference to susceptibility [11]. Although great advances have been made in researching the genetic mechanisms of plant disease resistance, the mechanisms underlying plant disease susceptibility remain unclear.

Populus deltoides possesses qualitative resistance to $M$. larici-populina [12]. Although hybrid vigor is generally high in these cultivars, most of the hybrids exhibit high rust susceptibility once the major resistance genes inherited from $P$. deltoides are defeated $[12,13]$. In a previous study, hybrid poplar $P$. nigra $\times P$. deltoides 'Robusta' was found to have non-race-specific resistance to $M$. larici-populina isolates collected from England [4], but the resistance was defeated by E4. Therefore, 'Robusta' provides suitable host material for studies of gene regulation involved in rust resistance/susceptibility.

MicroRNAs (miRNAs) play important roles in plant post-transcriptional gene regulation by repressing the translation of target mRNAs or targeting them for cleavage [14]. In plants, miRNAs regulate diverse processes, such as development [12], abiotic stress tolerance [15], and biotic stress [16]. Several studies have indicated that miRNAs play critical roles in disease resistance or susceptibility responses [16]. For example, several plant miRNAs, such as miR160, miR167, miR398 and miR1885, are responsive to biotic stress during both resistance and susceptibility interactions [17-19]. Additionally, in susceptible poplar, all fungi-responsive miRNAs have been found to be up-regulated in response to the infection of a canker pathogen [20].

The genome sequences of both poplar ( $P$. trichocarpa) and its rust pathogen $M$. larici-populina have now been published [21]. Thus, the poplar-poplar rust pathogen system is the only system in which the genome sequences of both tree host and rust pathogen are available. Although poplar genomic analyses have revealed the genetic potential of susceptibility and transcriptomics has allowed for the deciphering of gene expression in space and time, little is known about the post-transcriptional regulation of resistance gene expression. Recent evidence has suggested that post-transcriptional silencing involving small RNAs of cellular rather than pathogenic origin might have broad implications in potentiating basal defense and race-specific resistance to microbes in plants [22]. The present study was conducted to decipher the regulatory functions of miRNA in a susceptible poplar and to unravel the complexity of post-transcriptional regulation of resistance genes in the susceptible poplar 'Robusta'.

\section{Results}

\section{Small RNA sequencing profile}

To study the post-transcriptional regulation associated with poplar susceptibility to rust, miRNA accumulation was investigated by small RNA sequencing. Two small RNA (sRNA) libraries constructed from a combination of seven selected time points of the 'Robusta' leaves of -rust (rust-free) and + rust (E4 rust-inoculated) were sequenced on an Illumina GAIIx system. To acquire more details of the sRNA background in one sequencing, seven time points covering the major developmental transitions of the urediniospores in poplar leaves were selected, consistently with previous studies [23, 24]. A total of 8,840,983 (Additional file 1: Figure S1 A) and 9,736,262 (Additional file 1: Figure $\mathrm{S} 1 \mathrm{~B})$ raw sequences were generated from the -rust and + rust libraries, respectively. Blast homology searches were performed against $M$. larici-populina (version 1.0, JGI website http://genome.jgi-psf.org/Mellp1/), and the mapped reads were removed from the + rust library.

The length distribution of the mappable reads showed that 15-nt reads (18\%) were the most abundant sequences in the + rust sRNA library and that 21-nt reads (17.5\%) were the second most abundant sequences (Additional file 1: Figure S2). In contrast, the -rust sRNA library showed that 21-nt reads were the most abundant sequences (Additional file 1: Figure S2), constituting $19.3 \%$ of all reads. This latter result is in agreement with results from sRNA studies of $P$. balsamifera [25] and Vitis vinifera [26], in which the 21-nt sRNAs showed the highest abundance. However, this result differs from the results of studies of other plants, in which 24-nt sRNAs were most abundant [27-28].

By removing the redundant sequences from mappable reads, 3,820 and 4,070 unique sequences were identified from the -rust and + rust libraries, respectively. After removing the consensus sequences, a total of 5,138 unique miRNAs were identified in these two sRNA libraries. 
These unique miRNAs were classified into two groups: known miRNAs and predicted miRNAs (Additional file 1: Figure S1 C and D, Additional file 1: Table S1). Known miRNAs were further divided into two subgroups: Gp1a and Gp1b (Additional file 1: Text S1). Predicted miRNAs were further divided into six subgroups: Gp2a, Gp2b, Gp3a, Gp3b, Gp4a and Gp4b (Additional file 1: Text S1). By removing the no-hairpin subgroups and the subgroups that did not map to the poplar genome, Gp1a, Gp1b, Gp2a and Gp4a were identified as candidate miRNA subgroups. Among these candidate miRNA subgroups, 1,474 and 1,475 miRNAs were obtained from the -rust and + rust sRNA libraries, respectively. The potential novel miRNAs identified by deep sequencing were deposited in The
European Nucleotide Archive (WEBIN ID number: Hx2000050201).

According to the eleven features of the miRNA hairpins (LC Sciences, Houston, TX, USA, Additional file 1: Text S2), the secondary structure of genes corresponding to the potential novel miRNAs (Gp4a) were identified (Table 1), and 4 predicted secondary structures of the potential novel miRNAs are shown as examples (Additional file 1: Figure S3).

\section{Identification of rust-responsive miRNA in $P$. nigra $\times P$. deltoides}

MiRNAs regulate gene expression in plants by promoting the degradation of or repressing the translation of target

Table 1 The potential novel miRNAs and their targets

\begin{tabular}{|c|c|c|c|c|}
\hline \multicolumn{3}{|l|}{ miRNA } & \multicolumn{2}{|l|}{ Targets } \\
\hline miRNA & $\log _{2}$ & Sequences $\left(5^{\prime}-3^{\prime}\right)$ & Annomination & $\log _{2}$ \\
\hline PC-5p-310376_3 & 12.64 & UUUUCAAUAAUUGCAUCAAUA & NB-ARC domain-containing disease resistance protein & -0.37 \\
\hline PC-3p-1638036_1 & 11.06 & UUUGAUAGAACCACUGCA & xyloglucan endotransglucosylase/hydrolase 28 & 0.38 \\
\hline PC-5p-674947_1 & 11.06 & GCAGUGACUUGAAAGAA & RING-box 1 & -0.00 \\
\hline PC-3p-2607690_1 & 2.77 & CGGCAACGGAAUUUAUUUUAUU & E3 ubiquitin ligase SCF complex subunit SKP1/ASK1 family protein & 0.03 \\
\hline PC-5p-236891_4 & 2.27 & UCUCAACGAAACUUCAAUCGU & Sec23/s protein transport family protein & -0.23 \\
\hline PC-3p-142486_6 & 1.77 & UUACACAGAACCAUGCC & Golgi nucleotide sugar transporter 4 & 0.17 \\
\hline PC-3p-2198351_1 & 1.18 & ACUCGUGAUUUUAACAACCUUGGU & RING/FYVE/PHD-type zinc finger family protein & -0.14 \\
\hline PC-3p-390998_2 & 1.18 & CUGGCAGGGAUUGUAACUGUG & HXXXD-type acyl-transferase family protein & 0.14 \\
\hline PC-3p-1014196_1 & 1.18 & CAAAGAAGCUGCAAUUUGAAA & LRR and NB-ARC domains-containing disease resistance protein & -0.54 \\
\hline PC-5p-86003_12 & 1.18 & CUUCUCCAUGGAGUGCAUG & Ferritin 2 & -0.49 \\
\hline PC-3p-193248_4 & 1.03 & CCUGGACACUGUUCACU & Polyketide cyclase/dehydrase and lipid transport superfamily protein & 0.08 \\
\hline PC-5p-275646_3 & -1.14 & CGAAGAGUUCUUGGUGUUUU & Preprotein translocase SecA family protein & -0.70 \\
\hline PC-5p-336387_2 & -1.40 & GUAGUUCUUUCCCAUCAAACC & Phosphoglycerate kinase 1 & -0.09 \\
\hline PC-3p-123255_8 & -1.40 & AAAUUGAUGAAUUUAUGGAGU & WRKY DNA-binding protein 3 & 0.06 \\
\hline PC-3p-401772_2 & -1.40 & UUGAGAUUGUAGUAGCAGUGAU & Jasmonate-zim-domain protein 1 & -1.29 \\
\hline PC-3p-1525453_1 & -10.88 & CUGCCCUCGAGAGACUC & Multidrug resistance-associated protein 6 & 0.14 \\
\hline PC-3p-2241919_1 & -10.88 & UGUGUUGUAGUAAUUACU & Chaperone protein htpG family protein & -1.10 \\
\hline PC-5p-1903277_1 & 0.18 & ATGTTATGTGGTAGATAA & Aldehyde dehydrogenase $3 \mathrm{H} 1$ & -1.02 \\
\hline PC-3p-1909651_1 & -9.88 & ACTCATAATGTTGCAAC & Glutathione S-transferase 6 & 0.54 \\
\hline PC-5p-359200_2 & -10.88 & TTACTCGATAATGATATGAAC & Cysteine-rich RLK (RECEPTOR-like protein kinase) 25 & 1.23 \\
\hline PC-5p-2592253_1 & -10.88 & AGATAAATCCAACGATACCAAAAA & Cysteine-rich RLK (RECEPTOR-like protein kinase) 42 & 1.02 \\
\hline PC-5p-1977684_1 & -9.88 & ATTCCACTGATTTCTTTTATGATG & Cysteine-rich RLK (RECEPTOR-like protein kinase) 26 & 1.12 \\
\hline PC-3p-719252_1 & -9.88 & GTTAATTTCACACACACACACAC & Cysteine-rich RLK (RECEPTOR-like protein kinase) 26 & 1.00 \\
\hline PC-5p-1490392_1 & -9.88 & TTTGTGAATTCCAAAAGTGGT & Cysteine-rich RLK (RECEPTOR-like protein kinase) 29 & 1.13 \\
\hline PC-5p-1501811_1 & -9.88 & ATTCCCTCGATATATACCGATAGA & Cysteine-rich RLK (RECEPTOR-like protein kinase) 34 & 1.81 \\
\hline PC-3p-2480743_1 & 0.18 & GAAGTGATCAACATAGAAATGATTT & Cysteine-rich RLK (RECEPTOR-like protein kinase) 42 & 8.64 \\
\hline PC-5p-1349045_1 & -9.88 & CATTTGTTCATGCTTCTTA & Disease resistance protein (CC-NBS-LRR class) family & -0.08 \\
\hline PC-5p-1150271_1 & 10.06 & TTGAATTAGAGAAATTAGGGT & SID2 & -0.19 \\
\hline PC-5p-1312398_1 & -10.88 & TGCCCCTTGAATGCCATTCTT & OPR3 & 0.92 \\
\hline PC-3p-272434_3 & -1.82 & AGACACACCAAAACTTCAATGATG & MPK4 & -0.19 \\
\hline
\end{tabular}


mRNAs, both transcriptionally and post-transcriptionally $[18,28]$. To associate rust fungi $M$. larici-populina infection with an alteration of poplar miRNA accumulation, the differential expression of miRNAs in the two libraries was compared using the $\log _{2^{-}}$(+rust/-rust) fold changes of the normalized miRNA copy numbers (Fig. 1a). $\log _{2^{-}}$(+rust/-rust) fold changes $>1$ were designated as indicating 'up-regulated miRNA', and changes $<-1$ were designated as indicating 'down-regulated miRNA' (Fig. 1b).

Analysis of the differential expression of 5,138 miRNAs showed that 2,126 (41.38\%) were expressed in both the -rust and + rust libraries (co-expression, Fig. 1c), whereas $1,335(25.98 \%)$ and 1,677 (32.64\%) were preferentially expressed in -rust and + rust libraries, respectively (Fig. 1c). Analysis of co-expressed miRNAs resulted in the identification of 541 miRNAs (out of 2,126; $25.45 \%$ ) that showed significant differential expression between the -rust and + rust libraries. Of these, $376(73.15 \%)$ were upregulated, and 165 (32.10\%) were down-regulated after the inoculation of rust.

In the four candidate miRNA subgroups, 1,081 miRNA members were responsive to rust infection ( $\log _{2}$-fold changes $>1$ or $<-1)$. Restricting the analysis to only those miRNAs with more than 5 raw reads in both of the two sRNA libraries revealed changes of only 61 miRNAs as shown in Fig. 2. In the subgroup Gp1a, 7 miRNAs were up-regulated and 7 miRNAs were down-regulated in response to rust infection (Fig. 2a). With the exception of 3 up-regulated miRNAs, no differentially expressed
miRNAs were found in the Gp1b subgroup (Fig. 2b). In the subgroup Gp2a, 5 miRNAs were up-regulated and 8 miRNAs were down-regulated in response to rust infection (Fig. 2c). In total, 12 miRNAs were up-regulated and 7 were down-regulated in response to rust infection in the Gp4a subgroup and were potentially novel miRNAs (Fig. 2d). The most robustly changed miRNA was PC5p-310376_3 (Gp4a, Fig. 2d), with a peak $\log _{2}$-fold change of 12.641 .

\section{Identification of miRNA targets}

To identify genes targeted by miRNAs, a degradome cDNA library from + rust poplar leaves was constructed. In total, 8,409,874 raw, short sequencing reads were obtained from this library (Additional file 1: Table S2), and 5,077,704 unique reads mapped to the Populus mRNA database (http://www.ncbi.nlm.nih.gov/nuccore/). A list of all of the confidently detected mRNA targets along with the corresponding alignments for the miRNAmRNA interactions were generated by the pipeline. All five categories (Additional file 1: Text S3) [29] of the sliced-target transcripts were found. According to the 1,475 miRNAs identified in the + rust sRNA library, 947 (64.20 \%) miRNAs potentially targeted to 8,463 poplar genes, and a total of 12,722 regulating interactions were found.

These targets included 31 plant defense genes that were regulated by 26 miRNA (Table 2), such as the disease resistance protein TIR-NBS-LRR, regulated by miR482a (Fig. 3f); the HXXXD-type acyl-transferase family protein
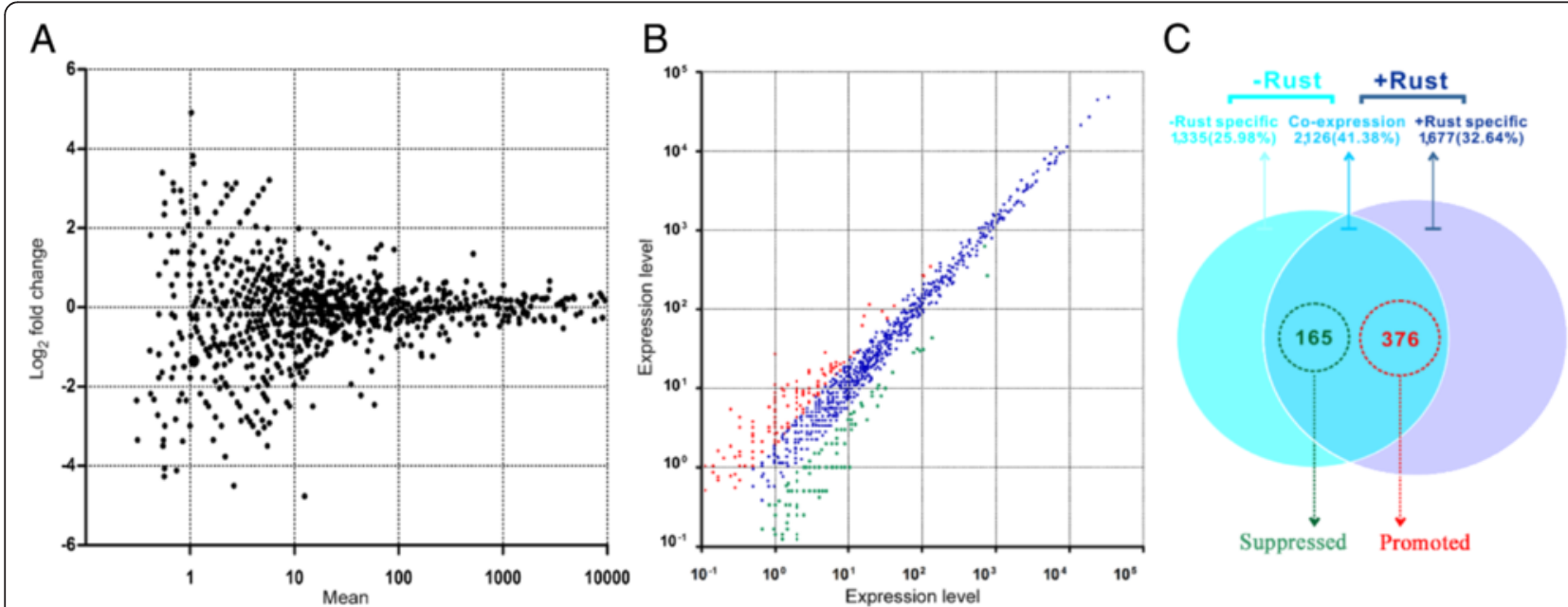

Fig. 1 Identification of rust-responsive miRNAs in P. nigra $\times P$. deltoides 'Robusta'. a Scatter plot of gene expression in + rust and -rust libraries according the $\log _{2}$ of normalized reads. Expression levels are normalized by the mean numbers of transcripts per million clean reads (TPM). $\mathbf{b}$ Scatter plot of miRNA expression levels according to the raw reads. Data points below (green) or above (red) the slope line represent down-regulated or up-regulated miRNAs. c Comparison of differentially expressed miRNAs between the -rust and + rust libraries. The Venn diagram displays the distribution of 5,138 unique miRNAs between -rust (left, cyan circle) and + rust (right, slate circle) libraries. The $\log _{2}-$ (+rust/-rust) fold changes $>1$ were designated as indicating 'up-regulated miRNAs', and the changes $<-1$ were designated as indicating 'down-regulated miRNAs' 

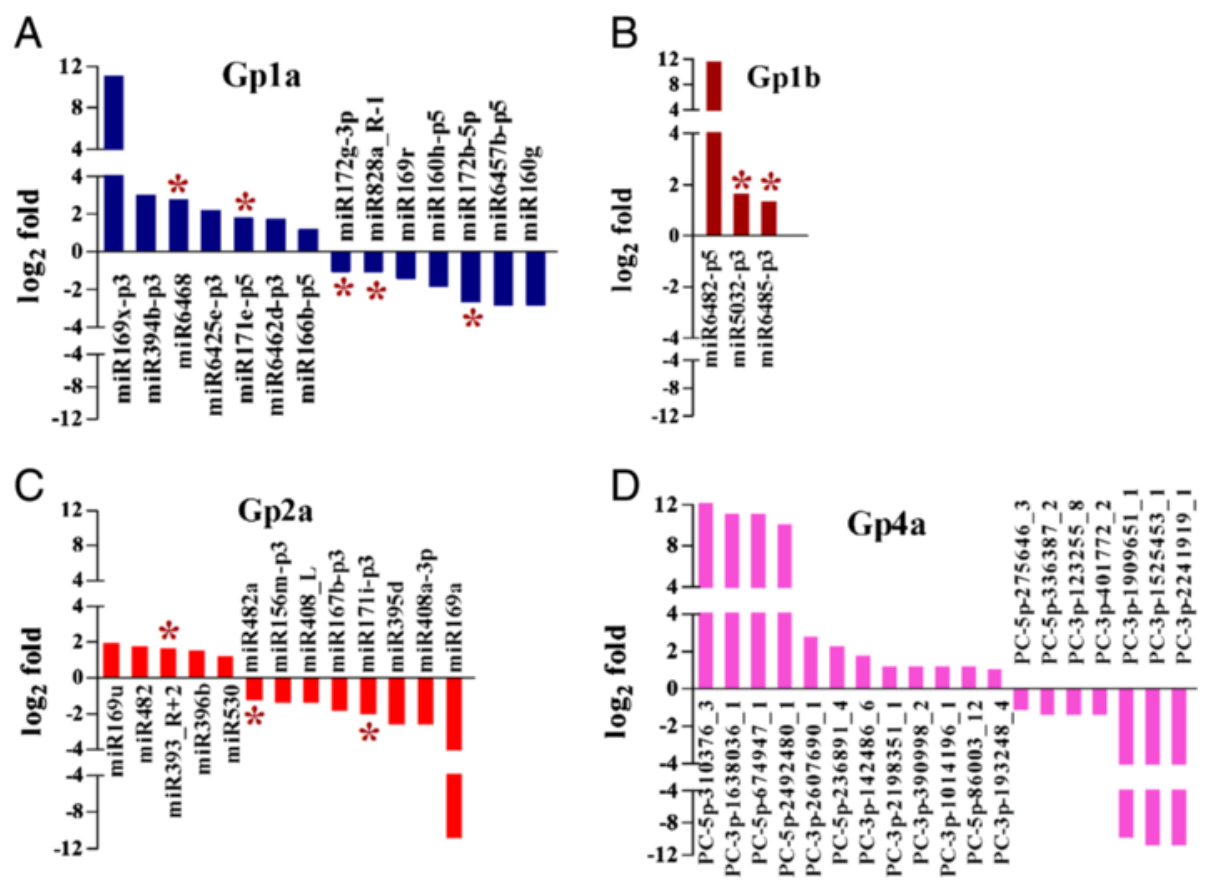

Fig. 2 Profiles of the differentially expressed miRNAs. a the differentially expressed Gp1a miRNAs; $\mathbf{b}$ the differentially expressed Gp1b miRNAs; c the differentially expressed Gp2a miRNAs; $\mathbf{d}$ the differentially expressed Gp4a miRNAs. Profiles of the differentially expressed Gp1a, Gp1b, Gp2a and Gp4a miRNAs responsive to rust fungi M. larici-populina infection identified in sRNA libraries from infected (+rust) and uninfected (-rust) P. nigra $\times$ P. deltoides 'Robusta' plants. Only miRNAs with more than 5 raw reads in both of the two libraries are shown. The positive and negative values indicate miRNAs whose expression was up-regulated and down-regulated by rust, respectively. The asterisks denote the expression of the miRNAs at different time points as tested by RT-qPCR

gene, regulated by miR6468 (Fig. 3j); and the woundresponsive family protein, regulated by miR394-p3. Additionally, these targets included 27 transcription factors that were regulated by 27 miRNAs (Additional file 1: Table S3), such as the AP2/ERF superfamily transcription factor gene, regulated by miR172g-3p (Fig. 3a); the GRAS family transcription factor gene, regulated by miR171i-p3 (Fig. $3 \mathrm{~b}$ ); the MYB transcription factor gene, regulated by miR828a (Fig. 3d); and the WRKY70 gene, regulated by miR172b-5p (Fig. 3e).

\section{Digital gene expression}

To characterize the target gene expression pattern, two digital gene expression (DGE) libraries of -rust and + rust were constructed and sequenced. There were 16,409,618 and 18,186,321 reads sequenced from the -rust and + rust libraries, respectively. More than $99 \%$ of the raw reads passed the filter cut-off, resulting in 18,117,197 and $16,338,785$ clean reads. In total, 73,013 unique genes were obtained, and 65,822 of those unique genes were mappable. Of the unique reads, $1,331,698(25.07 \%)$ and $1,419,523(24.85 \%)$ sense reads were mapped to one gene for the -rust and + rust libraries, respectively. The remaining $73.38 \%$ of the unique reads for the -rust library and the remaining $73.60 \%$ for the + rust library matched to multiple genes. The remaining reads were unique antisense or both sense and antisense reads that were mappable to genes.

To compare the differential expression patterns between the -rust and + rust libraries, we normalized (by the means of TPM) the read distribution for the gene expression level in each library to construct an effective library size and determined the significance of differentially expressed transcripts with $x^{2}(P \leq 0.01)$. According to the annotation of these genes, the rust infection-regulated gene expression was analyzed (Fig. 4).

According to the results of the DGE analysis, the targets of the potential novel miRNAs with altered expression were analyzed (Tables 1 and Additional file 1: S4). Then, the $\log _{2}$-fold changes of plant defense-related genes and their regulating miRNAs were analyzed (Table 2).

On the basis of differential expression analysis [30] and control of the false discovery rate (FDR) [31], 282 differentially expressed target genes were identified. To compare these results with the real-time quantitative polymerase chain reaction (RT-qPCR) results, the $\log _{2^{-}}$ (+rust/-rust) fold changes $>1$ were designated as indicating 'up-regulated genes', and the changes $<-1$ were designated as indicating 'down-regulated genes'.

Gene Ontology (GO) analysis was performed for all of the differentially expressed genes (Additional file 1: Table S5). Altogether, 282 differentially expressed target 
Table 2 Popular defense related genes responsive to rust infection and their regulating miRNA

\begin{tabular}{|c|c|c|c|c|}
\hline \multicolumn{2}{|l|}{ Targets } & \multicolumn{3}{|l|}{ miRNA } \\
\hline Annomation & $\log _{2}$ & miRNA & Group & $\log _{2}$ \\
\hline A20/AN1-like zinc finger family protein & 1.16 & miR855-p5 & Gp3a & -0.4 \\
\hline Aldehyde dehydrogenase $3 \mathrm{H} 1$ & -1.02 & PC-5p-1903277_1 & Gp4a & 0.18 \\
\hline ARM repeat superfamily protein & 2.55 & miR172j-p3 & Gp3b & 10.06 \\
\hline Auxin response factor 16 & 1.06 & miR160e-5p & Gp1a & 0.25 \\
\hline BED zinc finger & 0.55 & miR858a-p3 & Gp3b & 10.06 \\
\hline Chaperone protein htpG family protein & -1.19 & PC-3p-2241919_1 & Gp4a & -10.88 \\
\hline Cysteine synthase D1 & 1.33 & miR167a-p3 & Gp3b & -9.88 \\
\hline Cytochrome P450, family 82, subfamily G, polypeptide 1 & 1.11 & miR482b-p5 & Gp3a & 11.06 \\
\hline Disease resistance protein (TIR-NBS-LRR class) & -0.94 & miR393b-p5 & Gp3b & -9.88 \\
\hline Dormancy/auxin associated family protein & -1.29 & miR396e-p3 & Gp2b & 10.06 \\
\hline Early nodulin-like protein 18 & 1.05 & miR824-p3 & Gp3a & 2.18 \\
\hline ENTH domain-containing protein & 0.54 & miR171e-p5 & Gp1a & 1.77 \\
\hline F-box family protein & -0.65 & miR394-p3 & Gp3b & 10.06 \\
\hline Glutaredoxin family protein & 0.58 & miR6300 & Gp2b & -0.82 \\
\hline Glutaredoxin family protein & 0.9 & miR2606b-p3 & Gp3a & 0.18 \\
\hline Glutathione S-transferase 6 & 0.54 & PC-3p-1909651_1 & Gp4a & -9.88 \\
\hline Histone superfamily protein & 1.37 & miR400-p5 & Gp3a & 11.64 \\
\hline Homeodomain-like superfamily protein & -1.01 & miR2655e-p3 & Gp3b & -10.14 \\
\hline HXXXD-type acyl-transferase family protein & 0.81 & miR6468 & Gp1a & -2.77 \\
\hline Jasmonate-zim-domain protein 1 & -2.48 & PC-3p-401772_2 & Gp4a & -1.4 \\
\hline Late embryogenesis abundant domain-containing protein/LEA domain-containing protein & 1.02 & miR394-p3 & Gp3b & 10.06 \\
\hline myo-inositol-1-phosphate synthase 2 & 1.29 & miR5072-p3 & Gp3a & 10.06 \\
\hline Pathogenesis-related 4 & -1.02 & miR5517-p5 & Gp3a & 1.18 \\
\hline P-loop containing nucleoside triphosphate hydrolases superfamily protein & 1.04 & miR394-p3 & Gp3b & 10.06 \\
\hline PR5-like receptor kinase & 0.5 & miR394-p3 & Gp3b & 10.06 \\
\hline Proton gradient regulation 5 & -1.04 & miR156g-p5 & Gp3b & -9.88 \\
\hline RING/FYVE/PHD zinc finger superfamily protein & 1.14 & miR824-p3 & Gp3a & 2.18 \\
\hline TIR-NBS-LRR16 & 1 & miR482a_2s & Gp2a & 1.18 \\
\hline Trichome birefringence-like 33 & 1.1 & miR5293-p5 & Gp3a & -0.18 \\
\hline Wound-responsive family protein & -1.57 & miR394-p3 & Gp3b & 10.06 \\
\hline WRKY DNA-binding protein 11 & 0.79 & miR2097-p3 & Gp3a & 10.06 \\
\hline
\end{tabular}

genes were linked to $56 \mathrm{GO}$ terms. Once the GO terms of interest were identified for the unigenes, the differentially expressed genes and their associated miRNAs were analyzed (Additional file 1: Text S5). From the GO terms, we found that many processes, such as response to stress (GO:0006950), heat shock protein binding (GO:0031072), unfolded protein binding (GO:0051082), oxidationreduction process(GO:0055114), were prominently downregulated and that the processes of response to oxidative stress (GO:0006979), metabolic process (GO:0008152), biosynthetic process (GO:0009058), and cell wall macromolecule catabolic process (GO:0016998) were up-regulated.
The impact of rust infection on post-transcriptional regulation in the susceptible poplar

From the analysis of the miRNA, degradome cDNA and DGE libraries, 12,722 regulatory interactions (947 miRNAs to 8,463 target genes) were identified (Fig. 5a). Among the 947 miRNAs involved, 250 down-regulated ( $\log _{2}$-fold changes $<1$ ) and 301 up-regulated ( $\log _{2}$-fold changes $>1$ ) rust-responsive miRNAs were associated with 4,099 and 4,781 regulatory interactions (Fig. 6), respectively. Among the 4,099 regulatory interactions targeted by 250 downregulated miRNAs, 59 (1.439\%) target genes were upregulated ( $\log _{2}$-fold changes $\left.>1\right)$, $61(1.488 \%)$ target genes were down-regulated $\left(\log _{2}\right.$-fold changes $\left.<-0.5\right), 3,967$ 


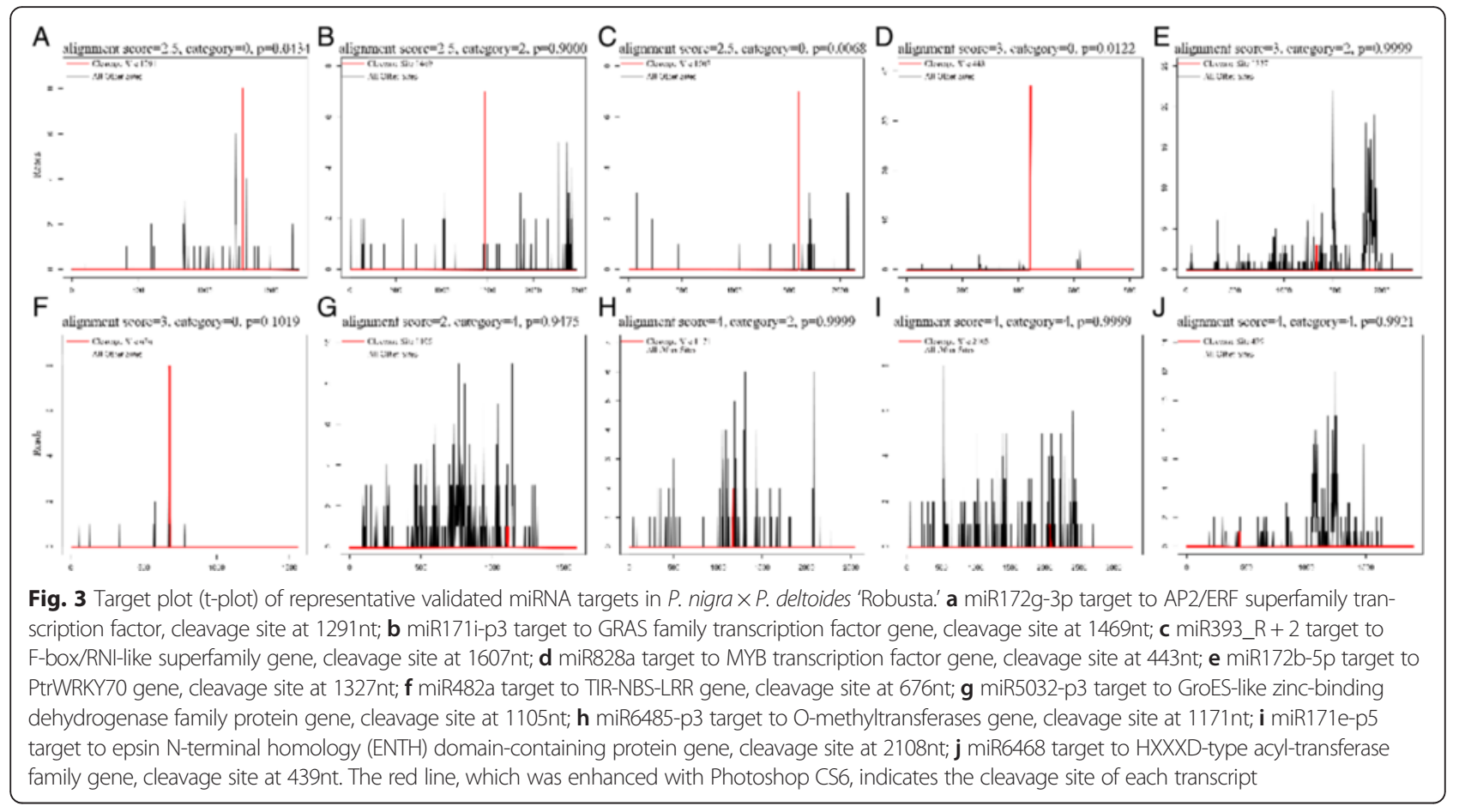

(96.77 \%) target genes were unchanged ( $\log _{2}$-fold changes $>-1$ and $<1)$, and the other target genes (12 regulating interactions) were undetected by DGE analysis. Among the 4,781 regulatory interactions targeted by 301 up-regulated miRNAs, 44 (0.92\%) target genes were down-regulated, 73 (1.53\%) target genes were up-regulated, 4,648 (97.22\%) target genes were unchanged, and the other target genes (16 regulating interactions) were undetected by DGE analysis. The resistance-related miRNAs and their target genes were
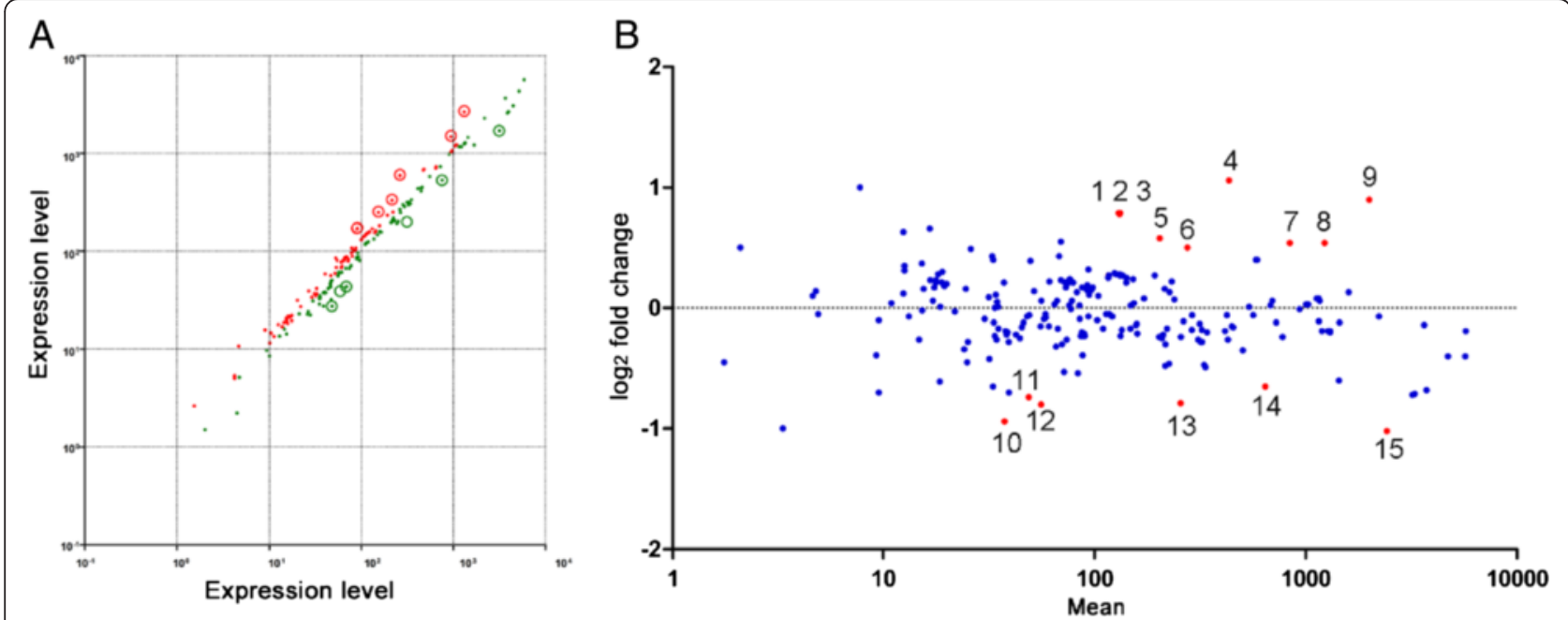

Fig. 4 Scatter plot of rust infection-regulated gene expression. a Scatter plot of mRNA expression levels according the raw reads. Data points above (green) or below (red) the slope line represent down-regulated or up-regulated miRNAs. Differentially expressed genes are denoted with circles based on $x^{2}(P \leq 0.01)$. $\mathbf{b}$ Scatter plot of gene expression in + rust and -rust libraries according the $\log _{2}$ of normalized reads. Expression levels are normalized to TPM. Differentially expressed genes are labeled red based on $x^{2}(P \leq 0.01)$ (details in Table 2). 1, 2 and 3: WRKY DNA-binding protein 11; 4: Auxin response factor 16; 5: Glutaredoxin family protein; 6: PR5-like receptor kinase; 7: epsin N-terminal homology (ENTH) domain-containing protein/clathrin assembly protein-related; 8: glutathione S-transferase 6; 9: Glutaredoxin family protein; 10: disease resistance protein (TIR-NBS-LRR class); 11: Histone H3 K4-specific methyltransferase SET7/9 family protein; 12: Disease resistance protein (TIR-NBS-LRR class); 13: HXXXD-type acyl-transferase family protein; 14: F-box family protein; 15: Pathogenesis-related 4 


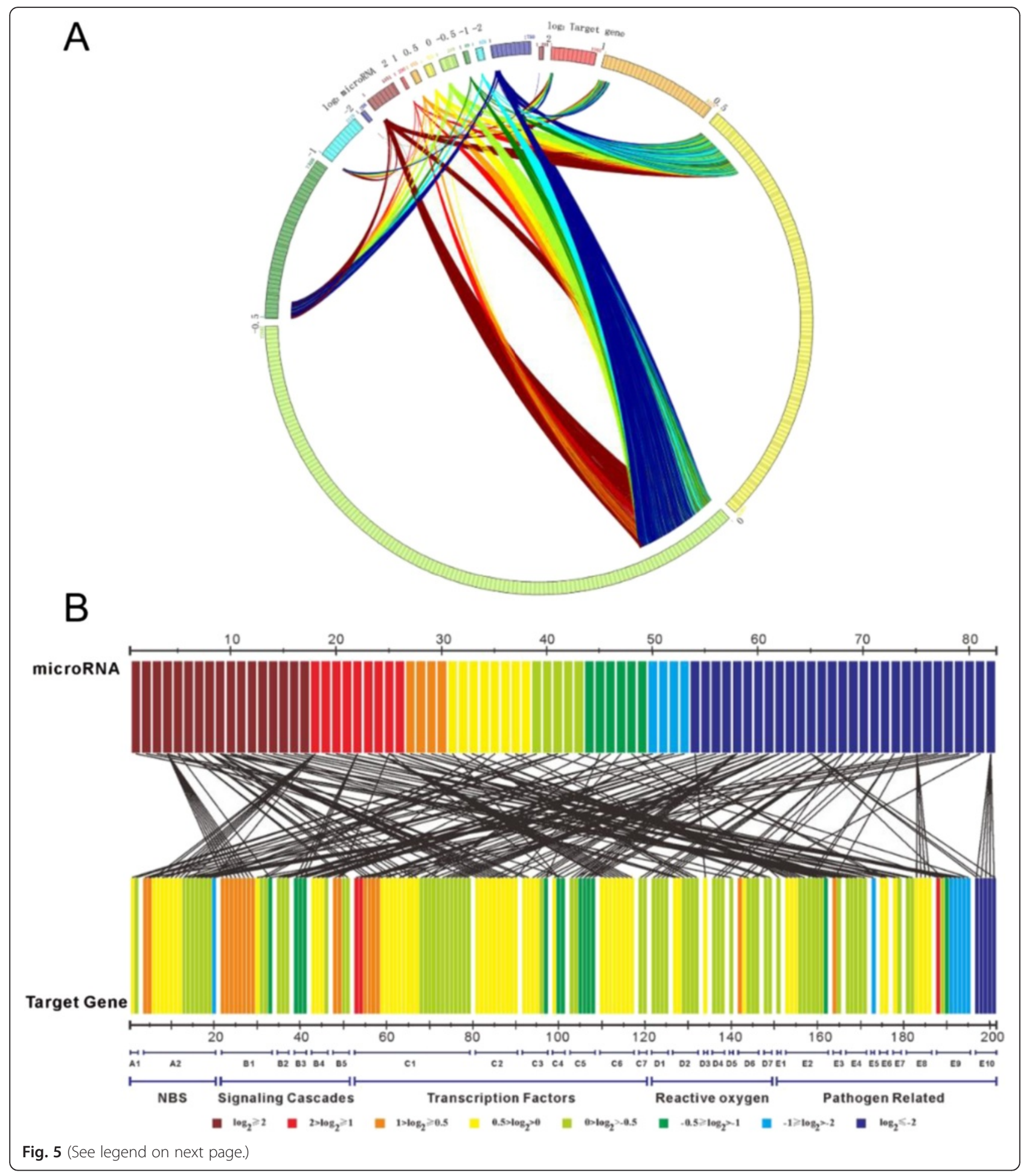


(See figure on previous page.)

Fig. 5 Post-transcriptional analyses of miRNAs and genes in response to infection by rust in 'Robusta'. a Post-transcriptional analyses of miRNAs and genes in response to infection by rust in the susceptible poplar. b Post-transcriptional analyses of miRNAs and resistance genes in response to infection by rust in the susceptible poplar. A1: CC-NBS-LRR; A2: TIR-NBS-LRR; B1: MAP kinase; B2: MAPKJERK kinase; B3: Kunitz family trypsin and protease inhibitor protein; B4: 2-cysteine peroxiredoxin; B5: Glutaredoxin family protein; C1: WRKY DNA-binding protein; C2: WRKY family transcription factor; C3: Basic-leucine zipper (bZIP) transcription factor family protein; C4: Ethylene responsive element binding factor; C5: MYB family transcription factor; C6: MYB-like HTH transcriptional regulator family protein; C7: Jasmonate-zim-domain protein; D1: Copper/Zinc superoxide dismutase; D2: Manganese superoxide dismutase; D3: Ascorbate peroxidase; D4: Monodehydroascorbate reductase; D5: Glutathione peroxidase; D6: Glutathione reductase; D7: Glutathione S-transferase; D8: Ferritin; E1: F-box and associated interaction domains-containing protein; E2: F-box family protein; E3: F-box/RNI-like superfamily protein; E4: Pathogenesis related homeodomain protein; E5: Pathogenesis-related protein; E6: Pathogenesis-related thaumatin superfamily protein; E7: Peptidoglycan-binding LysM domain-containing protein; E8: Phospholipase; E9: Wound-responsive family protein; E10:

Wound-responsive protein-related

found in poplar 'Robusta', but not all of those miRNAs and their target genes were in compliance with this negative regulation (Additional file 1: Figure S4, Additional file 1: Table S4, transcription model M2b).

Furthermore, we analyzed the post-transcriptional regulation of 82 miRNAs to 171 resistance genes, such as the genes encoding intracellular nucleotide-bindingsite (NBS) -leucine-rich repeat (LRR) proteins (Fig. 5b, A1 and A2), signaling cascades (Fig. 5b, B1 to B5), transcription factors (Fig. 5b, C1 to $\mathrm{C} 7$ ), reactive oxygen (Fig. 5b, D1 to D7) and pathogenesis-related (Fig. 5b, E1 to E10) (Additional file 1: Table S4). Out of these 82 miRNAs, 28 (34 \%) regulated only one target gene, whereas the other $54(66 \%)$ regulated more than one target gene. In contrast, 132 (77 \%) resistance genes were regulated by one miRNA, whereas the remaining genes $(23 \%)$ were regulated by more than one miRNA.

Altogether, 226 resistance regulating interactions were identified, and these regulating interactions were further divided into five regulation modes. The first mode included 73 regulating interactions (32\%). The expression of target genes was unchanged (Additional file 1: Table S4, regulation mode M1b) or up-regulated (Additional file 1: Table S4, regulation mode M1a) in the first mode, although the accumulation of miRNA was increased. Only 9 regulating interactions $(4 \%)$ were identified in the second mode. In this mode, the accumulation of miRNA was unchanged, but the expression of target genes was up-regulated (Additional file 1: Table S4, regulation mode M2a) or strongly suppressed (Additional file 1: Table S4, regulation mode M2c). The maximum amount of regulating interactions, a total of 89 interactions (39\%), was identified in the third mode. In this mode, the accumulation of miRNA was decreased, but the expression of target genes was down-regulated (Additional file 1: Table S4, regulation mode M3c) or unchanged (Additional file 1: Table S4, regulation mode M3b). The fourth mode included 31 regulating interactions (14\%, Additional file 1: Table S4, regulation mode M2b), and both the miRNA and the target gene were unresponsive to the rust

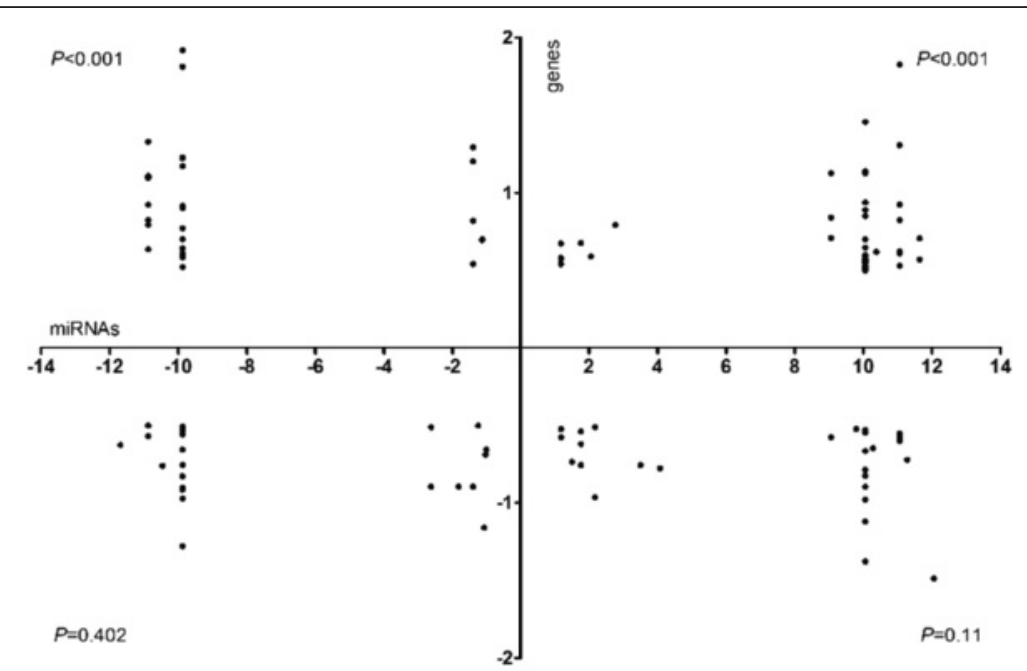

Fig. 6 Targets of rust-responsive miRNAs among rust-regulated genes. The $x$-axis indicates the $\log _{2^{-}}$(+rust/-rust) transformed fold change of rust-responsive miRNAs; $\log _{2}$ - (+rust/-rust) fold changes $>1$ were designated as indicating 'up-regulated miRNAs', and changes $<-1$ were designated as indicating 'down-regulatedmiRNAs'. The $y$-axis indicates the $\log _{2}$-transformed fold change of rust-regulated expressed genes; the $\log _{2^{-}}$(+rust/-rust) fold changes $>1$ were designated as indicating 'up-regulated target genes', and changes $<-1$ were designated as 'down-regulatedtarget genes'. The $P$-value was assessed by a 2-tailed $x^{2}$ test 
infection. The last mode, which is consistent with the classical post-transcriptional regulation theory, included 24 regulating interactions ( $11 \%)$. In the last mode, the accumulation of miRNA and the expression of target genes were in compliance with negative correlation (Additional file 1: Table S4, regulation modes M1c and M3a).

\section{Validation and temporal pattern of miRNA expression and target genes}

To avoid missing significant differential changes in the mixed samples sequencing, RT-qPCR was performed for 10 differentially expressed miRNAs $\left(\log _{2}>1\right.$ or $\left.<-1\right)$ and target genes at $2 \mathrm{~h}$ post inoculation (hpi) of rust, $6 \mathrm{hpi}$, $12 \mathrm{hpi}, 1$ day post inoculation (dpi), $2 \mathrm{dpi}, 4 \mathrm{dpi}$ and 7 dpi. These miRNAs were selected based on principles Additional file 1: Text S5 and are listed in Additional file 1: Table S6. The expression patterns of the RT-qPCR results showed details that had not been found in the sRNA libraries and DGE sequencing.

According to previous studies, before $1 \mathrm{dpi}$, infection hyphae extend into the mesophyll and differentiate into the first haustorial structures [32, 33]. Then, biotrophic growth continues, and the fungal biomass greatly increases between 2 and 4 dpi $[34,35]$. During this time period, the expression of miRNA volatility continually changes over time, and we found 5 expression patterns related to the development of rust occurring on poplar leaves (Additional file 1: Figure S5; $\alpha, \beta, \gamma, \delta$ and $\varepsilon$ patterns, Additional file 1: Text S6). The expression of targets (Additional file 1: Figure S6) did not accompany changes in miRNA for all 7 time periods (Fig. 7), but several negative interactions were found for the $\alpha$-pattern miRNA (miR172b-5p targeting the WRKY70 gene, miR171i-p3 targeting the GRAS family transcription factor gene, miR393_R + 2 targeting the F-box/RNI-like superfamily gene, miR828a targeting the MYB transcription factor gene and miR172b-5p targeting the WRKY70 gene) and the $\gamma$-pattern miRNA (miR171e-p5 targeting the epsin N-terminal homology domain-containing protein gene) at $1 \mathrm{dpi}$; in these cases, the targets were up-regulated, whereas the miRNAs were down-regulated (Fig. 7, boxed with red dashed lines).

\section{Framework of the susceptible poplar defense signal pathway} The miRNA and target global maps indicated that most of the related KEGG (Kyoto Encyclopedia of Genes and Genomes) pathways (Additional file 1: Figure S7) were down-regulated or up-regulated after rust infection, whereas few target genes were responsive to rust infection in the susceptible poplar. The other pathways, such as plant hormone signal transduction, cell cycle and plant-pathogen interaction, showed the same pattern; i.e., the miRNAs reacted strongly to the infection, but the target genes reacted mildly. The pathway of Plant-pathogen Interaction indicated that the miRNAs related to fungal pathogen- associated molecular patterns (PAMPs, including reactive oxygen species), Hypersensitive response (HR) and fungal effectors were responsive to rust infection. However, only 4 of 31 genes were up-regulated in the same pathway.

According to the regulatory interactions, an miRNAmediated susceptible poplar defense signal pathway related to rust infection was revealed (Additional file 1: Table S7). The receptor-like kinase genes $R L K 25, R L K$ 26, $R L K 29, R L K 34$ and $R L K 42$ were up-regulated, whereas the regulating miRNAs were down-regulated by rust infection. $R L K 14$ was up-regulated, but the regulating miRNAs remained unchanged. $R L K 3$ was up-regulated, as were the regulating miRNAs up-regulated.

The NBS-LRR family of resistance (R) proteins induced effector-triggered susceptibility (ETS). The results of degradome cDNA library sequencing indicated that 25 TIRNBS-LRR family genes were regulated by 9 miRNAs. Although 7 miRNAs were down-regulated significantly, none of the corresponding 18 target genes were up-regulated. Two miRNAs were up-regulated, whereas the target TIRNBS-LRR family genes were unchanged. Only 1 TIR-NBSLRR gene family was up-regulated after the infection of rust fungi, whereas the regulating miRNA was unchanged. Additionally, 4 unchanging CC-NBS-LRR family genes were regulated by 2 down-regulated miRNAs. The suppression of the TIR-NBS-LRR-regulating miRNA indicated that recognition occurred between the rust effectors and the poplar $\mathrm{R}$ proteins but that the signal promoting the expression of $\mathrm{R}$ proteins was blocked. This process explains why 'Robusta' cannot prevent rust infection at the ETS stage.

Many downstream defense signal pathways were also found. First, pathogenesis-related (PR) genes, which were triggered by effector signals and transcription factors, were investigated (Additional file 1: Table S7). MiR5517-p5 was up-regulated, and its target PR gene was suppressed. Two pairs of PR genes and their regulating miRNA were unchanged. MiR3447-p3 was strongly up-regulated, yet its target PR gene was unchanged.

Second, the pathway related to salicylic acid (SA), which is the main signal molecule for systemic acquired resistance (SAR) and $\mathrm{HR}$, did not respond to rust infection, although many of the regulating miRNAs were found to be down-regulated or up-regulated after infection. The enhanced disease susceptibility 1 gene (EDS1, regulated by the promotion of miR3434-p3) and the phytoalexin-deficient 4 gene (PAD4, regulated by the suppression of miR4248b-p3), which act to induce genes upstream of SA accumulation, did not respond to rust infection. Ankyrin-repeat protein NPR1, which acts downstream of SA and promotes the expression of the SARassociated genes PR-1, BGL2, and PR-5 in Arabidopsis [36], also did not respond to rust infection. The expression of the 'Robusta' enhanced disease susceptibility 5 (EDS5) gene was up-regulated slightly (it is also regulated by the 


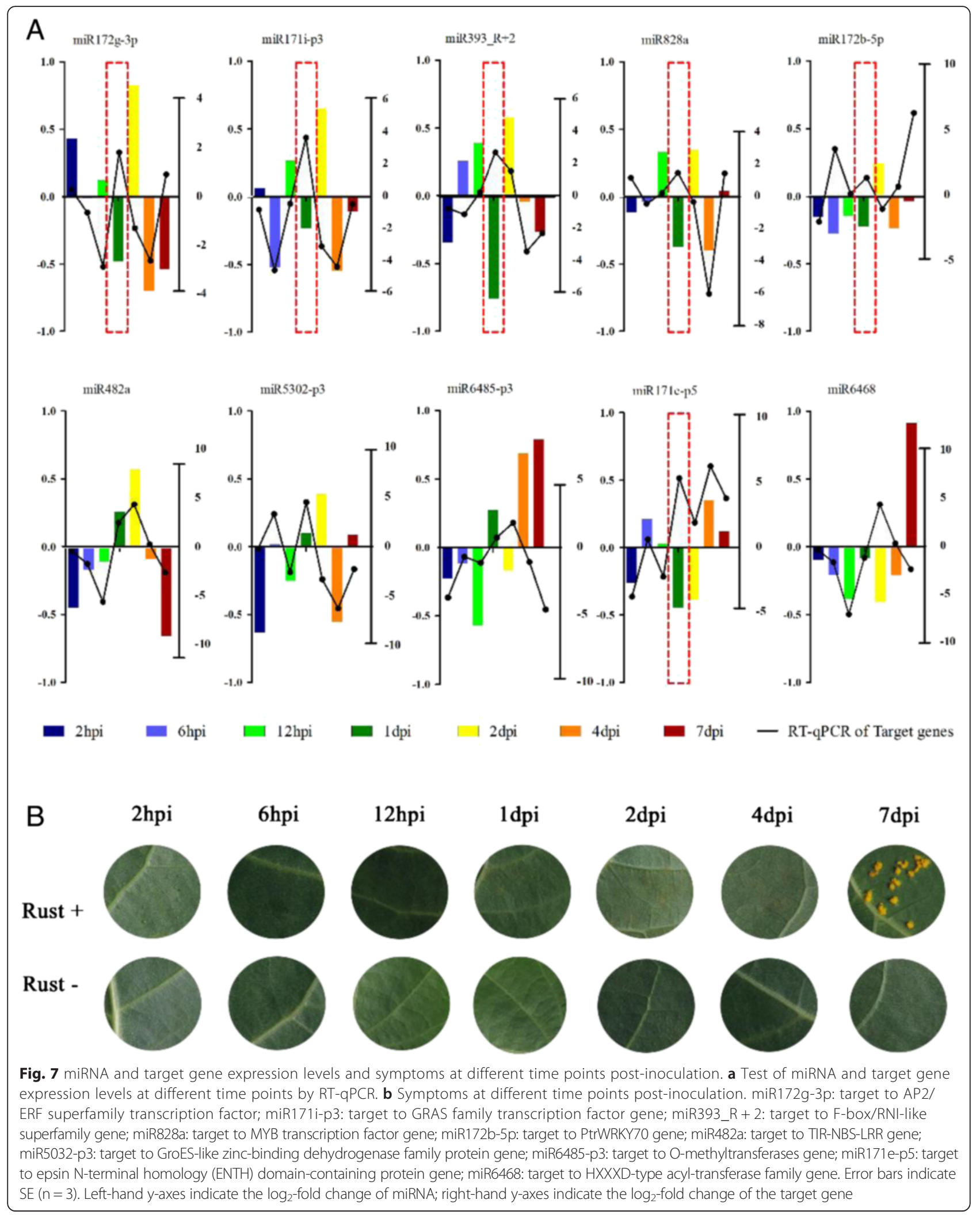


suppression of miR4248b-p3), whereas SA inductiondeficient 2 (SID2) was unchanged (regulated by the upregulated PC-5p-1150271_1) after rust infection, although EDS5 and SID2 function upstream of SA accumulation in Arabidopsis [37]. The programmed cell death-related gene also did not respond to rust infection. These results suggest that the signal transduction pathways are blocked, which typically leads to an HR-related accumulation of SA and programmed cell death to halt pathogen invasion. Third, with the exception of the suppression of mitogenactivated protein kinase 4 (MPK4, regulated by the downre down-regulated PC-3p-272434_3) down-regulated and the promotion of 12-oxpophytodienoic acid reductase 3 (OPR3, regulated by the down-regulated PC-5p1312398_1) up-regulated, no genes related to jasmonic acid (JA) or ethylene (ET) responded to rust infection, including coronatine-insensitive protein 1 (COI1, undetected), jasmonic acid-amido synthetase 1 (JAR1, regulated by the down-regulated miR393b-p5_1) and Ethylene-insensitive protein 2 (EIN2, undetected). Normally, JA- and ET-dependent resistance is up-regulated by lesion formation in A. thaliana. MPK4, COI1 and JAR1 are required to transduce the JA signal, whereas EIN2 is required to transduce the ET signal [36].

\section{Discussion}

The miRNA-mediated post-transcriptional regulation of plant resistance to biotic stress has been described in several plant pathosystems [22, 36-41]. In contrast, the molecular mechanisms of plant susceptibility to biotic stress and the post-transcriptional regulation of such susceptibility are poorly studied. Identifying the genetic basis of host susceptibility/resistance is a prerequisite for understanding the interaction between pathogen and plant host. Based on miRNA, degradome cDNA and DGE library analyses, we investigated the regulatory functions of miRNA in a susceptible poplar under rust attack. We found that the genes involved in the miRNAmediated post-transcriptional regulation of the defense signal pathway were inactivated after infection by E4 in 'Robusta', whereas this regulation was functional during E1 infection. This inactivation was the major characteristic of 'Robusta' susceptibility.

The small RNA sequencing revealed a significant peak in abundance of 15 -nt reads, with 835 unique 15-nt miRNAs were found in the + rust sRNA library. Moreover, 120 miRNAs targeted 113 unigenes, which included many disease-related genes, such as the AP2/B3 transcription factor protein family, the disease resistance CC-NBS-LRR class protein family, the disease resistance TIR-NBS-LRR class protein family, cellulose synthase 6 genes, LRR and NB-ARC domain-containing diseaseresistance protein genes and stress-inducible protein genes. However, unexpectedly, none of these disease-related target genes responded to rust infection. These results indicate that the 15-nt miRNAs responded to rust infection and showed a significant increase but that they failed to regulate their target genes in the susceptible poplar.

Basal defense and $R$ gene-mediated resistance are the two branches of the defense response carried out by plants under pathogen stress [42]. Basal defense marks the first line of defense and consists of a set of defined receptors referred to as pattern recognition receptors (PRRs), which recognize conserved, slowly evolving MAMPs or PAMPs [44]. The receptor-like kinases, which are part of the initial stages of PAMPs and PTI, were up-regulated in this compatible (susceptible poplar vs. virulent rust) pathosystem, and the miRNAs related to these stages were responsive to rust infection. These results indicate that the poplar defense commences with pathogen-associated molecular patterns (PAMPs) and PAMP-triggered immunity (PTI) and that the miRNAs are still functional at this stage.

The second branch of the defense response acts largely inside the cell and uses the polymorphic NB-LRR protein products encoded mostly by $R$ genes [44]. Although the basal defense of the poplar was almost completely destroyed in this compatible pathosystem, $R$ genemediated resistance was not fully functional. Moreover, the post-transcriptional regulation of the $R$ gene did not occur. Only 1 TIR-NBS-LRR gene family, regulated by miR472b, was up-regulated after rust fungi infection, although poplar genomes contain 64 TIR-NBS-LRR and 119 CC-NBS-LRR immune receptors encoded by $R$ genes that recognize the pathogen infection and trigger resistance responses [45].

Twelve miRNAs that guide the cleavage of 4 CC-NBS$L R R$ and 25 TIR-NBS-LRR transcripts were identified (Additional file 1: Table S7). Two TIR-NBS-LRR and 2 CC-NBS-LRR genes were regulated by miRNAs that belong to the mir482 superfamily; this result is consistent with those from previous studies [46, 47]. Both miR482b and its target CC-NBS-LRR were unresponsive to rust infection. In contrast, although miR482 was up-regulated ( $\log _{2}$-fold change $\left.=1.77\right), 6$ TIR-NBS-LRR target genes were unchanged ( $\log _{2}$-fold change values ranging from 0.04 to 0.49 ). As one of the most important plant immune system branches [4], NBS-LRR-mediated disease resistance is effective against obligate biotrophic pathogens and hemi-biotrophic pathogens [48]. Our results showed that the susceptible poplar NBS-LRR-related genes were not up-regulated by the rust biotrophic pathogen. Interestingly, another study showed that NBS-LRRs are not substantially induced by an incompatible rust strain in $P$. trichocarpa $\times P$. deltoides [45]. These findings suggest that the lack of substantial induction of NBS-LRRs by infection is not a characteristic specific to susceptible poplar but is common to both susceptible and resistant poplars. 
All of the signaling component genes, including the genes related to $E T, S A, J A$ and $H R$, were unresponsive to rust infection, which suggests that the regulation of miRNA-based HR signaling was blocked. Normally, plants produce phytohormones to cope with infection by diverse pathogens [49]. SA is involved in resistance to biotrophic pathogens, whereas JA and ET mediate resistance primarily to necrotrophic pathogens [50]. These results are consistent with our expectations and the genefor-gene hypothesis, because the heterozygous resistant $(R r)$ poplar and the homozygous virulent (avr) rust were used in this study. Most of the studied plant pathosystems are related to the $R$-Avr gene-mediated disease response during pathogen invasion [42]. However, in our study, the plant pathosystem of susceptible poplar vs. virulent rust (an $R$-avr gene-mediated disease response) provides a new perspective for understanding plant resistance/susceptibility. However, our findings provide only initial insight into this topic, and a great effort will be required for a full understanding of the post-transcriptional regulation related to plant resistance/susceptibility.

\section{Conclusions}

The results of the sRNA, degradome cDNA and DGE library analyses indicated that the defense-related posttranscriptional regulation of the susceptible poplar 'Robusta' only function normally during the initial stages of PAMPs and PTI. More importantly, the miRNA-mediated post-transcriptional regulation of defense signal pathway genes were inactivated after infection by virulent rust at the stage of effector-triggered susceptibility and at the subsequent stages of salicylic acid and HR response. This inactivation is the major characteristic of 'Robusta' susceptibility.

\section{Methods}

\section{Rust isolates}

The filial generation of virulent E4 and avirulent E1 was used in this study. Rust-infected poplar leaves were collected from $P$. trichocarpa cv. trichobelat Markington (northern England) and Alice Holt, Surrey (southern England). Rust isolates were derived from single uredinial pustules according previously reported methods [4]. The rust spores were stored at $-20{ }^{\circ} \mathrm{C}$.

\section{Plant materials and inoculation procedure}

The 1-year-old dormant cuttings of the hybrid poplar $(P$. nigra $\times P$. deltoides) 'Robusta' were used as a source of plant tissue. 'Robusta' was chosen because a previous study has found that this cultivar showed non-racespecific resistance to $M$. larici-populina isolates collected from England [4].

'Robusta' plants were grown in pots containing a sandpeat $(50: 50, \mathrm{v} / \mathrm{v})$ mixture and were watered daily with deionized water under $16-\mathrm{h} / 8$-h photoperiod in greenhouse conditions and $60-70 \%$ relative humidity for 10 weeks. At the time of inoculation, young trees were $70-100 \mathrm{~cm}$ tall and bore 10 to 14 fully expanded leaves.

The inoculation procedure was performed as described by Pei et al. [4] with some modifications. In brief, fully expanded leaves from leaf plastochrony index (LPI) 5 to 9 were detached from 'Robusta' plants and sprayinoculated on their abaxial surface with a rust spore suspension in deionized water containing $0.004 \%$ Tween 20 (1 drop in $100 \mathrm{ml}$ ) adjusted to 100,000 spores $\mathrm{ml}^{-1}$, or with deionized water containing $0.004 \%$ Tween 20 as a control. After inoculation, the inoculated leaves were incubated in a growth chamber at $16{ }^{\circ} \mathrm{C}$ with $16 \mathrm{~h}^{\text {day }}{ }^{-1}$ illumination $\left(80 \mathrm{uE} \mathrm{m}^{-2} \mathrm{~s}^{-1}\right)$ for various durations. Each treatment was replicated three times. The samples harvested at different time points $(2 \mathrm{hpi}, 6 \mathrm{hpi}, 12 \mathrm{hpi}$, $1 \mathrm{dpi}, 2 \mathrm{dpi}, 4 \mathrm{dpi}$, and $7 \mathrm{dpi}$ ) in the different treatments were then immediately snap-frozen in liquid nitrogen and maintained at $-80^{\circ} \mathrm{C}$ for further nucleic acid isolation.

\section{Total RNA preparation}

Total RNA was extracted from frozen leaves of 'Robusta' with the CTAB method. Two sets of total RNA were prepared, with one derived from the original RNA pool prepared from E4 rust-inoculated leaves (+rust) at the 2 hpi, 6 hpi, $12 \mathrm{hpi}, 1 \mathrm{dpi}, 2 \mathrm{dpi}, 4 \mathrm{dpi}$, and $7 \mathrm{dpi}$ time points and the other from the RNA pool derived from rust-free leaves (-rust, the control) at the same time points.

\section{sRNA, degradome CDNA and DGE library preparation and sequencing}

Two sRNA libraries were generated from the -rust and + rust RNA pools using the Illumina TruSeq Small RNA Preparation Kit according to Illumina's TruSeq Small RNA Sample Preparation Guide 1.

The degradome cDNA library of + rust was prepared following procedures previously described (Additional file 1: Text S7) [48,51-53]. Two DGE libraries representing -rust and + rust were prepared according to the Illumina/ Solexa standard protocol (directional mRNASeq sample preparation part \#15018460 Rev. A, October 2010. Illumina, San Diego, CA, USA) (Additional file 1: Text S8).

The purified sRNA, degradome cDNA and DGE libraries were used for cluster generation on Illumina's Cluster Station and then sequenced on the Illumina GAIIx platform by LC Sciences (Houston, TX, USA). To analyze the differential expression, three biological replicates were performed with the sRNA, degradome cDNA and DGE libraries. Each sample of degradome cDNA was sequenced for 80 cycles on one lane of the Illumina GAIIx platform (Illumina, San Diego, CA, USA) with the $2 \times 100 \mathrm{bp}$ module. DGE libraries were sequenced using the $1 \times 36$ bp module. 
Raw sRNA library sequencing reads were obtained using Illumina's Sequencing Control Studio software version 2.8 (SCS v2.8) following real-time sequencing image analysis and base-calling by Illumina's Real-Time Analysis version 1.8.70 (RTA v1.8.70).

Raw sequencing reads of the degradome cDNA library were obtained using Illumina's Pipeline version 1.5 software following sequencing image analysis by the Pipeline Firecrest Module and base-calling by the Pipeline Bustard Module.

\section{Bioinformatics analysis}

A proprietary pipeline script, ACGT101-miR version 4.2 (LC Sciences, Houston, TX, USA), was used for the sRNA library sequencing data analysis to obtain mappable sequences from raw sequencing data and to map the miRNA-mappable unique sequences to pre-miRNA, mature miRNA or genome.

A global normalization was used to correct sRNA copy numbers among the -rust and + rust libraries (Additional file 1: Text S10). The differential gene expression of the DGE libraries was analyzed according to the method of Simon Anders, [30].

\section{RT-qPCR of mature miRNAs}

RT-qPCR was performed with the One Step PrimeScript miRNA cDNA Synthesis Kit and the SYBR Premix ExTag II reagent kit (TaKaRa, Dalian, China) using the Stratagene Mx3000P qPCR system (Agilent, USA) to validate the expression levels of miRNAs after the rust inoculation at $2 \mathrm{hpi}, 6 \mathrm{hpi}, 12 \mathrm{hpi}, 1 \mathrm{dpi}, 2 \mathrm{dpi}, 4 \mathrm{dpi}$ and $7 \mathrm{dpi}$. All of the primers used in this study are listed in Additional file 1: Table S8. The 5.8S ribosomal RNA was used as the internal control [54]. This experiment was performed on three biological replicates. Quantification of the RT-qPCR results was conducted as previously described [55]. To compare the RT-qPCR results with the results of the high-throughput sequencing, the RT-qPCR results were normalized as $\log _{2^{-}}$(+rust miRNA copy/-rust miRNA copy) fold changes.

\section{Availability of supporting data}

All the supporting data are included as additional files as "Additional file 1".

\section{Additional file}

Additional file 1: Text S1. Unique miRNA classification. Text S2. Eleven features of miRNAs hairpin. Text S3. Five categories of the sliced-target transcripts. Text S4. Gene Ontology (GO) analysis. Text S5. Principles of the miRNA selected for RT-qPCR. Text S6. The 5 expression patterns of miRNA volatility change related to the development of rust. Text S7. Methods of + rust degradome cDNA libraries construction. Text S8. Detailed methods of RNAseq libraries construction. Text S9. The functions of CleaveLand 3.0 used in this study. Text S10. Procedure of a modified global normalization. Figure S1. Pie plots of data filtering and database mapping of the sRNA libraries from P. nigra $\times$ deltoids infected (+rust) and uninfected (-rust) with rust fungi $M$. larici-populina. Figure S2. Length distribution of mappable reads in SRNA libraries from $P$. nigra $\times$ deltoids infected (+rust) and uninfected (-rust) with rust fungi M. larici-populina. Figure S3. Predicted secondary structures of potential novel miRNAs from $P$. nigra $\times$ deltoids. Sequences indicated in red correspond to predicted miRNA. Figure S4. Targets of rust-responsive miRNAs among rust-regulated expressed genes. Figure S5. miRNA expression level validated by RT-qPCR at different time points post inoculation. Figure $\mathbf{S 6}$. Target gene expression level validated by RT-qPCR at different time points post inoculation. Figure S7. KEGG pathway and atlas. Table S1. A summary of standard data analysis results of the sRNA libraries from P. nigra $\times$ deltoids 'Robusta' infected (+rust) and uninfected (-rust) with rust fungi $M$. larici-populina. Table S2. Data summary of degradome library. Table S3. Transcription factors and their regulating miRNA. Table S4. post-transcriptional analyses of miRNA and resistance genes response to the infection of rust in 'Robusta'. Table S5. The Gene Ontology of the rust-responsive gene and their regulating miRNA. Table S6. miRNA and target genes expression level and symptoms at different time points post inoculation. Table S7. Poplar defense pathway miRNA and target genes. Table S8. Primers used in the RT-qPCR. (DOC $3467 \mathrm{~kb})$

\section{Abbreviations}

avr: Homozygous virulent; COl1: Coronatine-insensitive protein 1; DGE: Digital gene expression; dpi: Days post inoculation; EDS1: Enhanced disease susceptibility 1; EDS5: Enhanced disease susceptibility 5; EIN2: Ethyleneinsensitive protein 2; ET: Ethylene; ETS: Effector-triggered susceptibility; GO: Gene Ontology; hpi: Hours post inoculation; HR: Hypersensitive response; JA: Jasmonic acid; JAR1: Jasmonic acid-amido synthetase 1; KEGG: Kyoto Encyclopedia of Genes and Genomes; LPI: Leaf plastochrony index; LRR: Leucine-richrepeat; miRNAs: MicroRNAs; NBS: Nucleotide-binding-site; PAD4: Phytoalexin-deficient 4; PAMPs: Pathogen-associated molecular patterns; PR: Pathogenesis-related; PRRs: Pattern recognition receptors; PTI: The PAMP-triggered immunity; R: Resistance proteins; Rr: Heterozygous resistant; RT-qPCR: Quantitative real time polymerase chain reaction; SA: Salicylic acid; SAR: Systemic acquired resistance; SRNA: small RNA.

\section{Competing interests}

The authors declare that they have no competing interests.

\section{Authors' contributions}

Conceived and designed the experiments: DL, FW and ZW. Performed the experiments: DL and FW. Analyzed the data: FW and DL. Contributed reagents/materials/analytical tools: $F W, D L, L Z, C W, Q C, C N, R Z, Y L$ and $B W$. Wrote the paper: FW, DL and QC. All authors have read and approved the manuscript.

\section{Acknowledgments}

We are grateful to Dr. Minghao Pei (Rothamsted Research, Harpenden, Hertfordshire, UK) for his helpful suggestions and critical reading of the manuscript.

\section{Financial source}

This work was supported by the National Natural Science Foundation of China $(31500524,31570637)$, the Fundamental Research Funds for the Central Universities (2572015EY02, DL13BA01 and 2572015CA09), the National High Technology Research and Development Program of China (863 Program) and the National Natural Science Foundation of China (31300571).

Received: 26 August 2015 Accepted: 10 December 2015

Published online: 15 January 2016

\section{References}

1. Van Kraayenoord CWS, Laundon GF, Spiers AG. Poplar rusts invade New Zealand. Plant Dis Reporter. 1974;58:423-7.

2. Newcombe G, Chastagner GA. First report of the Eurasian poplar leaf rust fungus, Melampsora larici-populina, in North America. Plant Dis. 1993;77:532-5. 
3. Pei MH, Shang YZ. Study on the leaf rust of cathay poplar caused by Melampsora larici-tremulae Kleb [in Chinese]. J Northeastern Forestry Institute. 1984;12:40-9.

4. Pei MH, Ruiz C, Bayon C, Hunter T, Lonsdale D. Pathogenic variation in poplar rust Melampsora larici-populina from England. Eur J Plant Pathol. 2005;111:147-55

5. Bean WJ. Trees and shrubs hardy in the British Isles (Vol. III, 8th edn) London: John Murray Ltd.; 1976.

6. Boyle J, Winjum J, Kavanagh K, Jensen E. Planted forests: contributions to the quest for sustainable societies. The Netherlands: Kluwer Academic Publishers; 1999. p. 90.

7. Steenackers M. Breeding poplars for rust resistance -recent advances. Communications of the Faculty of Agricultural Sciences of the State University of Ghent, Belgium. 1988; 53:417-22

8. Steenackers V. Nouvelle race physiologique de Melampsora laricipopulina en Belgique (communication provisoire). In: FAO/CIP, 22e Réunion de Groupe de Travail des Maladies. Italy: Casale Monferrato; 1982. p. 6-10.

9. Pinon J, van Dam BC, Genetet I, De Kam M. Two pathogenic races of Melampsora larici-populina in north-western Europe. Eur J Forest Pathol. 1987;17:47-53.

10. Steenackers $M$, Steenackers $V$, Delporte $T$. A new race of M. laricipopulina in Belgium. In: International Poplar Commission, Turkey: Izmit. 1994.p.3-7.

11. Nancy AE. Plant disease susceptibility genes? Plant Cell. 2002;14:1983-6.

12. Dowkiw $A$, Jorge $V$, Villar $M$, Voisin $E$,Guérin $V$, Faivre-Rampant $P$, et al. Breeding poplars with durable resistance to Melampsora larici-populina leaf rust: a multidisciplinary approach to understand and delay pathogen adaptation. In: Sniezko RA, Yanchuk AD, Kliejunas JT, et al., editors. Proceedings of the fourth international workshop on the genetics of hostparasite interactions in forestry: Disease and insect resistance in forest trees. Albany: Department of Agriculture; 2012. p. 3-40.

13. Baohong Z, Xiaoping P, Cobb GP, Anderson TA. Plant microRNA: A small regulatory molecule with big impact. Dev Biol. 2006;289:3-6.

14. Jones-Rhoades MWW, Bartel DPP, Bartel B. MicroRNAs and their regulatory roles in plants. Ann Rev Plant Biol. 2006;57:19-53.

15. Jones-Rhoades MWW, David PB. Computational identification of plant microRNAs and their targets, including a stress-induced miRNA. Mol Cell. 2004;14:787-99.

16. Padmanabhan $\mathrm{C}$, Zhang $\mathrm{X}$, Jin $\mathrm{H}$. Host small RNAs are big contributors to plant innate immunity. Curr Opin Plant Biol. 2009;12:465-72.

17. Fahlgren N, Howell MD, Kasschau KD, Chapman EJ, Sullivan CM, Cumbie JS, et al. High-throughput sequencing of Arabidopsis microRNAs: evidence for frequent birth and death of MIRNA genes. PLoS One. 2007; 2:e219. doi:10.1371/journal.pone.0000219.

18. Jagadeeswaran G, Saini A, Sunkar R. Biotic and abiotic stress down-regulate miR398 expression in Arabidopsis. Planta. 2009;229:1009-14.

19. He XF, Fang YY, Feng L, Guo HS. Characterization of conserved and novel microRNAs and their targets, including a TuMV-induced TIR-NBSLRR class $R$ gene-derived novel miRNA in Brassica. FEBS Lett. 2008;582: 2445-52.

20. Zhao JP, Jiang XL, Zhang BY, Su XH. Involvement of microRNA-mediated gene expression regulation in the pathological development of stem canker disease in Populus trichocarpa. PLoS One. 2012;9:e44968. doi:10.1371/journal.pone.0044968.

21. Petre B, Morin E, Tisserant E, Hacquard S, Da Silva C, Poulain J, et al. RNAseq of early-infected poplar leaves by the rust pathogen Melampsora laricipopulina uncovers PtSultr3;5, a fungal-induced host sulfate transporter. PLoS One. 2012;8:e44408. doi:10.1371/journal.pone.0044408.

22. Olivier V. Post-transcriptional RNA, silencing in plant-microbe interactions: a touch of robustness and versatility. Curr Opin Plant Biol. 2008;11:464-70.

23. Hacquard S, Delaruelle C, Legué V, Tisserant E, Kohler A, Frey $P$, et al. Laser capture microdissection of uredinia formed by Melampsora larici-populina revealed a transcriptional switch between biotrophy and sporulation. Mol PlantMicrobe Interact. 2010;23:1275-86.

24. Duplessis S, Hacquard S, Delaruelle C, et al. Melampsora larici-populinatranscript profilingduring germination and timecourse infection of poplar leaves reveals dynamic expression patternsassociated with virulence and biotrophy. Mol Plant-Microbe Interact. 2011;7:808-18.

25. Abdelali B, Phillip KW, Scott DL, Claude WD, John EC. Conservation and divergence of microRNAs in Populus. BMC Genomics. 2007;8:481.
26. Vitantonio P, Gyorgy S, Simon M, Laura M, Vincent M, Tamas D, et al. dentification of grapevine microRNAs and their targets using highthroughput sequencing and degradome analysis. Plant J. 2010;62:960-76.

27. Rajagopalan R, Vaucheret $H$, Trejo J, Bartel DP. A diverse and evolutionarily fluid set of microRNAs in Arabidopsis thaliana. Genes Dev. 2006;20:3407-25.

28. Moxon S, Jing R, Szittya G, Schwach F, Rusholme PRL, Moulton V, et al. Deep sequencing of tomato short RNAs identifies microRNAs targeting genes involved in fruit ripening. Genome Res. 2008;18:1602-9.

29. Jinghua $Y$, Xunyan $L$, Baochen $X, N a$ Z, Xiaodong $Y$, Mingfang $Z$. Identification of miRNAs and their targets using high-throughput sequencing and degradome analysis in cytoplasmic male-sterile and its maintainer fertile lines of brassica juncea. BMC Genomics. 2013;4:9.

30. Simon A, Wolfgang $H$. Differential expression analysis for sequence count data. Genome Biol. 2010;11:R106.

31. By Yoav B, Daniel Y. The control of the false discovery rate in multiple testing under dependency. Annals Statistics. 2001;29:1165-88.

32. Stéphane $H$, Benjamin P, Pascal F, Arnaud H, Nicolas R, Sébastien D. The poplar-poplar rust interaction: insights from genomics and transcriptomics. Journal of Pathogens. 2011;2011:1-11

33. Laurans F, Pilate $\mathrm{G}$. Histological aspects of a hyper sensitive response in poplar to Melampsora larici-populina. Phytopathology. 1999;89:233-8.

34. Rinaldi C, Kohler A, Frey P, Duchaussoy F, Ningre N, Couloux A, et al. Transcript profiling of poplar leaves upon infection with compatible and incompatible strains of the foliar rust Melampsora larici-populina. Plant Physiol. 2007;144:347-66.

35. Hacquard S, Veneault-Fourrey C, Delaruelle C, Frey P, Martin F, Duplessis S. Validation of Melampsora larici-populina reference genes for in planta RTquantitative PCR expression profiling during time-course infection of poplar leaves. Physiol Mol Plant Pathol. 2011;75:106-12.

36. Glazebrook J. Genes controlling expression of defense responses in Arabidopsis - 2001 status. Curr Opin Plant Biol. 2001;4:301-8.

37. Nawrath C, Metraux JP. Salicylic acid induction-deficient mutants of Arabidopsis express PR-2 and PR-5 and accumulate high levels of camalexin. Plant Cell. 1999;11:1393-404.

38. Navarro L, Dunoyer P, Jay F, Arnold B, Dharmasiri N, Estelle M, et al. A plant miRNA contributes to antibacterial resistance by repressing auxin signaling. Science. 2006;312:436-9.

39. Monk CE, Hutvagner G, Arthur JSC. Regulation of miRNA transcription in macrophages in response to Candida albicans. PLoS One. 2010;10:e13669.

40. Liu PT, Wheelwright M, Teles R, Komisopoulou E, Edfeldt K, Ferguson B, et al. MicroRNA-21 targets the vitamin D-dependent antimicrobial pathway in leprosy. Nat Med. 2012;18:267-73.

41. Ma F, Xu S, Liu X, Zhang Q, Xu X, Liu M et al. The microRNA miR-29 controls innate and adaptive immune responses to intracellular bacterial infection by targeting interferon-gamma. Nat Immunol. 2011;12:861-9.

42. Om PG, Pradeep S, Raj KG, Indu S. Current status on role of miRNAs during plant fungus interaction. Physiol Mol Plant Pathol. 2014;85:1-7.

43. Jonathan DGJ, Jeffery LD. The plant immune system. Nature. 2006;444:323-9.

44. Dangl JL, Jones JDG. Plant pathogens and integrated defence responses to infection. Nature. 2001;411:826-33.

45. Annegret K, Cécile R, Sébastien D, Marie B, Danny G, Frédéric D, et al. Genome-wide identification of NBS resistance genes in Populus trichocarpa. Plant MolBiol. 2008;66:619-36.

46. Padubidri VS, Ho-Ming C, Kanu P, Donna MB, Bruno AS, David CB. A microRNA superfamily regulates nucleotide binding site-leucine-rich repeats and other mRNAs. Plant Cell. 2012;3:859-74.

47. Zhu QH, Fan L, Liu Y, Xu H, Llewellyn D, Wilson I. miR482 regulation of NBS-LRR defense genes during fungal pathogen infection in cotton. PLoS One. 2013;12: e84390. doi:10.1371/journal.pone.0084390.

48. Glazebrook J. Contrasting mechanisms of defense against biotrophic and necrotrophic pathogens. Annu Rev Phytopathol. 2005:43:205-27.

49. Robert-Seilaniantz A, Navarro L, Bari R, Jones JDG. Pathological hormone imbalances. Curr Opin Plant Biol. 2007;10:372e9.

50. Grant MR, Jones JDG. Hormone (dis) hormonymoulds plant health and disease. Science. 2009;324:750e2.

51. Zhaorong M, Ceyda C, Michael JA. Arabidopsis lyrata small RNAs: transient MIRNA and small interfering RNA loci within the Arabidopsis genus. Plant Cell. 2010:22:1090-103.

52. Addo-Quaye C, Eshoo TW, Bartel DP, Axtell MJ. Endogenous siRNA and miRNA targets identified by sequencing of the Arabidopsis degradome. Curr Biol. 2008;18:758-62. 
53. Addo-Quaye C, Miller W, Axtell MJ. CleaveLand: a pipeline for using degradome data to find cleaved small RNA targets. Bioinformatics. 2009;1:130-1.

54. Lu S, Sun YH. Chiang VL Stress-responsive microRNAs in Populus. Plant J. 2008:1:131-51.

55. Peng S, Dan L, Zhoujia Z, Weilun Y, Xinli X. Identification of droughtresponsive and novel Populus trichocarpa microRNAs by high-throughput sequencing and their targets using degradome analysis. BMC Genomics. 2013;14:233.

Submit your next manuscript to BioMed Central and we will help you at every step:

- We accept pre-submission inquiries

- Our selector tool helps you to find the most relevant journal

- We provide round the clock customer support

- Convenient online submission

- Thorough peer review

- Inclusion in PubMed and all major indexing services

- Maximum visibility for your research

Submit your manuscript at www.biomedcentral.com/submit 